\title{
Monitoring the Dynamics of Heterogeneous Catalysts by Electron Microscopy
}

\author{
Ramzi Farra, Ali Rinaldi, Mark Greiner, Jing Cao, Robert Schlögl, Marc-G Willinger
}

Fritz Haber Institute of the Max Planck Society, Department of Inorganic Chemistry, Berlin, Germany

It is known that the shape of metal catalysts adapts to the chemical potential of the surrounding atmosphere and that the active surface evolves dynamically under reaction conditions [1-3]. Different photon-based characterization techniques were improved and implemented to probe the active state of catalysts in situ. However, such techniques lack the spatial resolution as they provide information averaged over a macroscopic scale, which is much larger than the catalytic active nanostructures.

In situ Scanning Electron Microscopy (SEM) and Transmission Electron Microscopy (TEM) have proven to be powerful techniques for the study of atmosphere and temperature induced morphological or compositional changes of catalysts at micrometer- to atomic resolution scale [4,5]. Furthermore, in situ SEM and TEM can be used as complementary methods from the point view of applicable pressure range and the obtainable resolution. Hence, pressure gap impact on the catalyst's activity can be assessed using these techniques. On the other hand, collective (SEM) and individual (TEM) phenomena on the surface of active catalysts in the micro- to nanometer scale can be compared and correlated to the reactivity.

In this contribution we present in situ TEM and SEM results of the catalytic oxidation of hydrogen on Copper. Aside from the fundamental importance of this catalytic system, the investigation of copper redox chemistry is of great importance for decisive future energy related catalytic processes as methanol synthesis or water gas shift reaction.

In situ SEM experiments were conducted in the chamber of a modified commercial environmental scanning electron microscope. The instrument is equipped with a home-built heating stage and attached to a gas feeding system and a mass spectrometer for product analysis.

In situ TEM experiments were carried out using a Protochips, Inc. gas flow holder equipped with an environmental cell (a nanoreactor). The holder is combined with a home built gas feeding and a mass spectrometer for gas analysis. The environmental TEM cell allows monitoring gas-solid interactions under relevant catalytic conditions.

Metal catalysts were prepared in situ by decomposing the catalyst precursor in an oxidative atmosphere and subsequent reduction in hydrogen (figure 1). As the resulting metal catalysts were exposed to a reductive gas mixture $\left(\mathrm{H}_{2}\right.$ in $\left.\mathrm{He}\right)$ that contains a little amount of molecular oxygen, the catalytic reaction starts to proceed, as proved by the gas analysis of products which shows a concomitant increase of water and decrease of oxygen MS-signals (figure 2). The catalyst at work is very dynamic and shows continuous and erratic morphological changes, provided that the Wulff construction of crystals was preserved most often over time. The interpretation of this dynamic behavior of catalysts under working conditions needs much further elaborated studies that can control or slow down the kinetics of the reactions, and hence make it possible to exploit other TEM analysis techniques as in situ EELS and diffraction. Nevertheless, these results may evoke strong debates about the assumptions that were published in the catalysis literature on the basis of TEM observations made in vacuum. 
In this contribution we will outline how the implementation of complementary in situ electron microscopy techniques can enrich our understanding of the dynamic behavior of the active catalysts.

\section{References:}

[1] P.L. Hansen, et al., Science, 2002, 295, 5562, 2053-2055

[2] T. Lunkenbein, et al., Angew. Chem.-Int. Ed. 2015, 54, 4544-4548.

[3] J. R. Jinschek, Chem. Commun. 2014, 50, 2696-2706.

[4] Z.-J. Wang, G., et al., ACS Nano 2015, 9, 1506-1519.

[5] S. B. Vendelbo, et al., Nat. Mater. 2014, 13, 884-890.

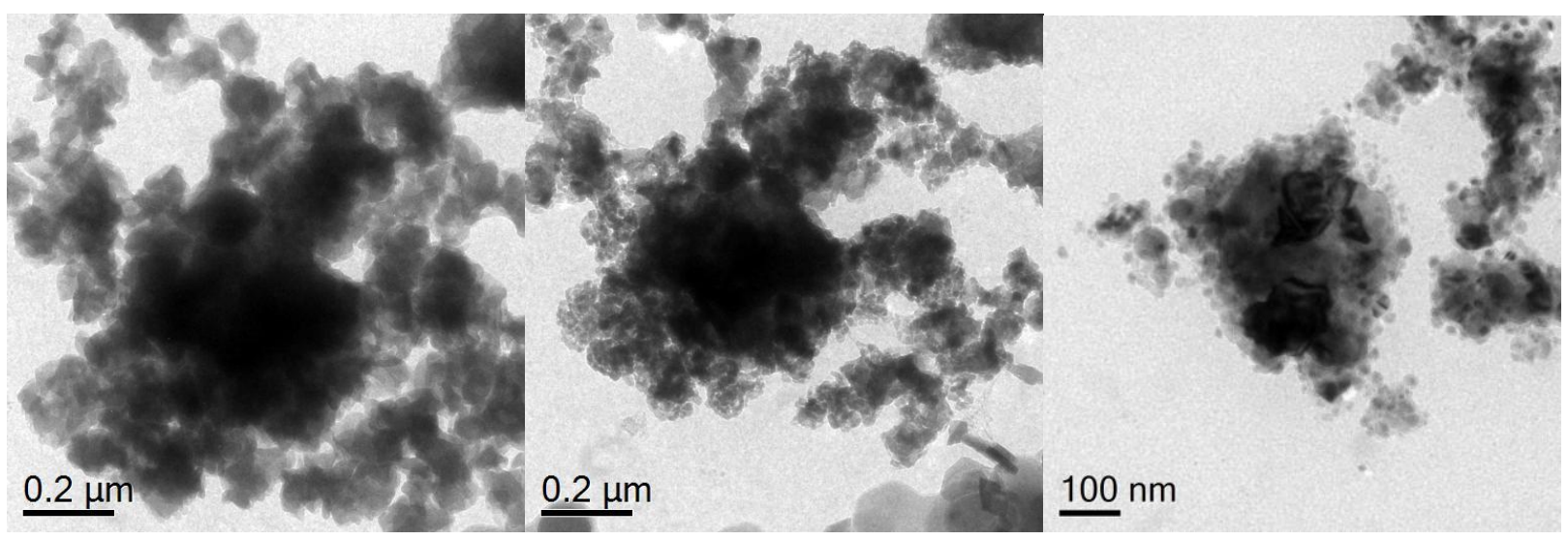

Figure 1. Sequential steps of catalyst preparation in situ (from left to right): Copper oxalate before oxidation, Copper oxide after calcination and Copper after reduction.
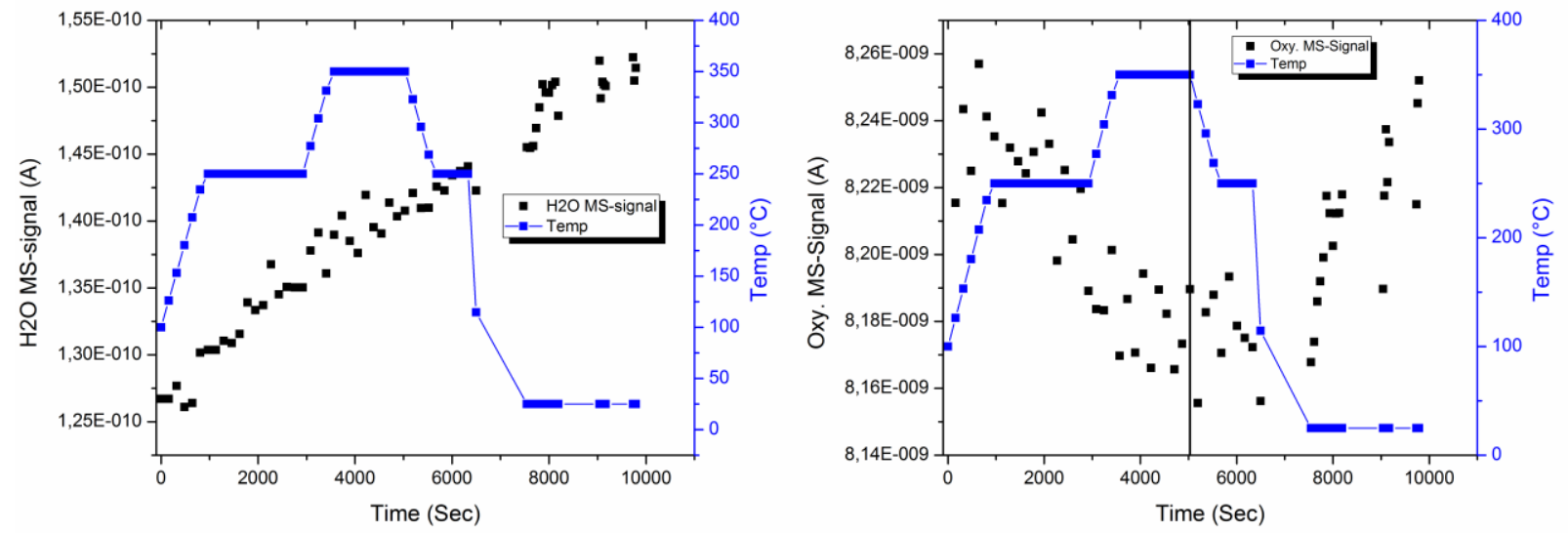

Figure 2. On-line mass spectroscopy signals of water (left) and Oxygen (right) at different reaction temperatures. Gas mixture flow was $0.1 \mathrm{ml} / \mathrm{min}$, pressure inside the nanoreactor was $200 \mathrm{mbar}$. 